\title{
Plant Diseases in Industrialized Countries
}

What we have seen so far perhaps gives you the wrong impression that serious epidemics only occur in developing countries or are something of the past. But it's not like that, and the story I'm about to tell you will confirm it quite effectively.

United States of America, 1970: we are in the famous "corn belt", a wide intensive corn cultivation area. At that time, American growers all cultivated some selected hybrids of corn, containing a cytoplasmic factor (so-called T factor, capable of maintaining male-sterility). Although very useful for hybridization work, this factor nevertheless caused a susceptibility towards a particular fungal race (T) of Heterosporium maydis (now called Bipolaris maydis), capable of producing a very selective toxin, T-toxin, active on corn.

That season was a disaster: the enormous selective pressure exerted by the cultivation of a few corn hybrids, containing the T-factor, over millions of acres, favoured the spread of the T-race pathogen. The damage was huge, as the disease affected the $80 \%$ of the cultivation area.

Obviously, being an epidemic of an annual species such as corn in an industrialized country in the 20th century, it caused mainly economic losses. Already the following year, the corn hybrids previously used, characterized by extreme susceptibility to the pathogen, were replaced. Crop failures, however, resulted in severe losses for corn growers.

The main culprit of this catastrophe was the cultivation on wide surfaces of very few hybrids with very similar genetic profiles. Genetic uniformity potentially exacerbates disease epidemics, a risk run every time that, on intensive cultivation areas, few crop varieties are grown on large surfaces. A typical risk of the most advanced agricultural systems. 